\title{
Middle cerebral artery territory infarction in a COVID-19 patient: a case report
}

\author{
Rizaldy Pinzon, ${ }^{1,2}$ Vincent Ongko Wijaya, ${ }^{1,2}$ Dessy Paramitha ${ }^{1,2}$
}

Check for updates

pISSN: 0853-1773 • elSSN: 2252-8083 https://doi.org/10.13181/mji.cr.204707 Med J Indones. 2021;30:66-70

Received: May 04, 2020

Accepted: September 02, 2020

Published online: November 14, 2020

Authors' affiliations:

${ }^{1}$ Duta Wacana Christian University School

of Medicine, Yogyakarta, Indonesia

${ }^{2}$ Bethesda Hospital, Yogyakarta,

Indonesia

Corresponding author:

Rizaldy Pinzon

Duta Wacana Christian University

School of Medicine, Jalan dr. Wahidin

Sudirohusodo No. 5-25, Yogyakarta

55224 , Indonesia

Tel/Fax: +62-274-563929 Ext. 606

E-mail: drpinzon17@gmail.com

\begin{abstract}
Coronavirus disease 2019 (COVID-19) mainly invades the respiratory system, yet previous reports have shown unusual manifestations of COVID-19, including cerebrovascular events. However, detailed case reports are still lacking. A 57-year-old male presented with sudden unconsciousness and right side lateralization for 6 hours before hospital admission. His test results were positive for severe acute respiratory syndrome coronavirus 2 infection. The head computed tomography (CT) scan showed infarction within the middle cerebral artery region. The scan was repeated 48 hours after admission and showed a massive infarct in the left hemisphere with hemorrhagic transformation. A chest CT showed the appearance of bronchopneumonia with ground-glass opacities in both lungs. The patient's condition was rapidly deteriorating, and he died on the third day after admission. Our findings suggest that ischemic vascular events may simultaneously develop due to the progression of COVID-19. A hypercoagulable state and vascular endothelial dysfunction have been proposed as complications of COVID-19 and are risk factors of thrombotic vascular events.
\end{abstract}

KEYWORDS case reports, COVID-19, ischemic stroke, middle cerebral artery
Coronavirus disease 2019 (COVID-19) is a newly emerging infectious disease and was first discovered at the end of 2019 as an outbreak of viral pneumonia in Wuhan, China. The disease has become a global pandemic causing a burden on healthcare and economics. ${ }^{1}$ The previous report has found that patients with severe disease were more likely to be admitted to an intensive care unit (ICU) and have poor outcomes. ${ }^{2}$ As of July 14, 2020, the severe acute respiratory syndrome coronavirus 2 (SARS-CoV-2) infection has confirmed in 78,572 cases and caused 3,710 deaths in Indonesia. ${ }^{3}$
Manifestations of COVID-19 range from typical symptoms, such as fever, headache, and cough, to lifethreatening conditions, including respiratory distress, pneumonia, multi-organ failure, and eventually death. ${ }^{2}$ Recent studies have suggested that COVID-19 has neuroinvasive abilities and presented its neurological manifestations, including cerebrovascular events, in patients infected with COVID-19..$^{2,4}$

The occlusion of the large middle cerebral artery (MCA) is a dangerous event with a high disability and mortality rate. However, its relationship with COVID-19 still lacks evidence and remains unclear. ${ }^{5}$ 
Although not well defined, the underlying mechanism could be related to increased inflammation and hypercoagulability due to COVID-19 progression. Studies have shown that most patients who died because of COVID-19 were associated with the progression of complications following the infection. .,6,7 $^{-1}$

This patient is a unique and challenging case of COVID-19. The patient presented with an acute and severe stroke that resulted in his rapid deterioration. Although the pathophysiology of the neurological manifestations caused by COVID-19 has not been studied in a detailed manner, the risk factors and possible mechanism of these manifestations need to be explored. ${ }^{7}$ Here, we report a patient with confirmed SARS-CoV-2 infection who presented with ischemic stroke within the MCA territory.

\section{CASE REPORT}

A 57-year-old man presented to the emergency department after a sudden onset of unconsciousness 6 hours before hospital admission. He had fever, fatigue, and nausea for 3 days before admission, but did not have cough or muscle pain symptoms at home. He previously had a dual antiplatelet treatment (100 mg aspirin and $75 \mathrm{mg}$ clopidogrel), $8 \mathrm{mg}$ candesartan, and $20 \mathrm{mg}$ atorvastatin for hypertension and ischemic heart disease for a year.

On admission, we performed a physical examination and found an impaired consciousness with the Glasgow coma scale (GCS) of $\mathrm{E}_{2} \mathrm{~V}_{2} \mathrm{M}_{3}$, conjugate deviation to the left side, isochoric pupils. His National Institute of Health Stroke Scale (NIHSS) score on admission was 30 (severe stroke). He also had hematemesis on admission. The patient was soon intubated and transferred to the ICU with a poor prognosis.

The patient had a history of close contact with positive COVID-19 patients 2 weeks before hospital admission. On admission, his rapid antibody test (IgM) results were positive. SARS-CoV-2 infection was also detected from the real-time polymerase chain reaction test from his nasopharyngeal swab sample. His chest computed tomography (CT) scan showed ground-glass opacities with interlobular thickening in both lungs, specifically in the posterior region associated with bronchopneumonia. (Figure 1). Therefore, the patient was diagnosed with COVID-19. Informed consent was

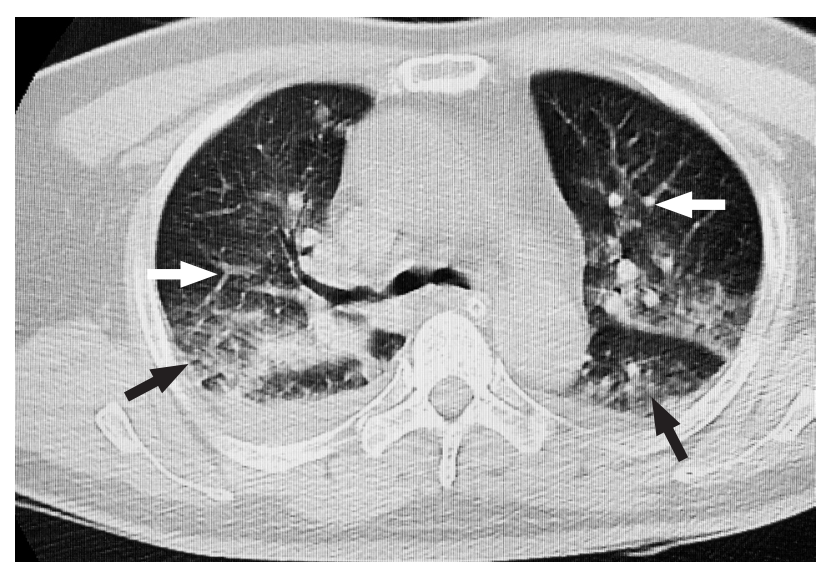

Figure 1. Axial chest computed tomography (CT) scan. Ground-glass opacities (black arrow) in both lungs and interlobular thickening (white arrow) indicating atypical bronchopneumonia

obtained from the patient's family for this report after his COVID-19 diagnosis.

His initial laboratory results were significant for elevated neutrophils (77.9\%), lymphopenia (16.5\%), and the neutrophil-to-lymphocyte ratio (NLR) was 5. His platelet levels were normal. However, the C-reactive protein (CRP) level was elevated to 26 $\mathrm{mg} / \mathrm{dl}$. D-dimer was not tested during hospital admission. His liver function test was also abnormal and showed elevated levels of alanine transaminase and aspartate transaminase to $249 \mathrm{u} / \mathrm{l}$ and $102 \mathrm{u} / \mathrm{l}$, respectively (Table 1 ).

Table 1. Laboratory parameters

\begin{tabular}{lcc}
\hline Parameter & Results & Reference \\
\hline Hemoglobin $(\mathrm{g} / \mathrm{dl})$ & 12.2 & $11.7-15.5$ \\
\hline Leucocyte $\left(\mathrm{x} 10^{3} / \mu \mathrm{l}\right)$ & 7.07 & $4.5-11.5$ \\
\hline Eosinophil $(\%)$ & 0.3 & $2-4$ \\
\hline Basophil $(\%)$ & 0.3 & $0-1$ \\
\hline Segment neutrophil $(\%)$ & 77.9 & $50-70$ \\
\hline Lymphocyte $(\%)$ & 16.5 & $18-42$ \\
\hline NLR & 5 & $1-3$ \\
\hline Monocyte $(\%)$ & 5.0 & $2-8$ \\
\hline Hematocrit $(\%)$ & 36.1 & $35.0-49.0$ \\
\hline Thrombocyte $\left(x 10^{3} / \mu l\right)$ & 190 & $150-450$ \\
\hline Natrium (mmol/l) & 136.3 & $136-146$ \\
\hline Potassium (mmol/l) & 3.49 & $3.5-5.1$ \\
\hline AST (u/l) & 102 & $0-55$ \\
\hline ALT (u/l) & 249 & $5.00-34.00$ \\
\hline Plasma CRP (mg/l) & 26 & $<5$ \\
\hline
\end{tabular}

NLR=neutrophil-lymphocyte ratio; $\mathrm{AST}=$ =aspartate aminotransferase; $\mathrm{ALT}=$ alanine aminotransferase; $\mathrm{CRP}=\mathrm{C}$-reactive protein 

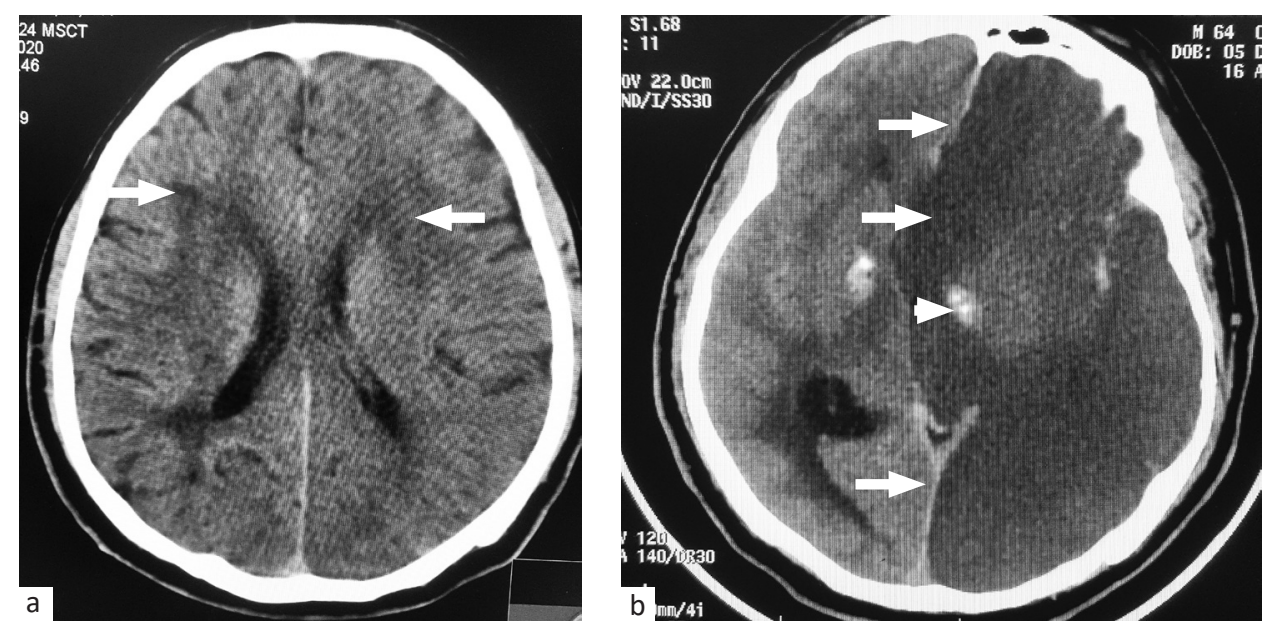

Figure 2. Brain computed tomography (CT) scan. (a) On admission showing hypodensity (arrow) in bilateral periventricular infarction within the middle cerebral artery (MCA) territory, (b) 48 hours after admission showing a massive cerebral infarction with a midline shift (arrow) in the left hemisphere and hemorrhagic transformation (arrowhead)

The first head CT scan on hospital admission showed bilateral periventricular infarction within the MCA territory (Figure 2a). The patient was treated with a proton pump inhibitor for the gastrointestinal bleeding and mannitol $1 \mathrm{~g} / \mathrm{kg} / 24$ hour as hyperosmolar therapy to reduce intracranial pressure. Hydroxychloroquine $500 \mathrm{mg}$ per 12 hour and azithromycin $600 \mathrm{mg}$ per 24 hour were administered as initial treatment of COVID-19. We repeated the $\mathrm{CT} 48$ hours after admission (Figure 2b). The scan showed a massive cerebral infarct in the left hemisphere with hemorrhagic transformation and a midline shift. A neurosurgical consultation was performed for the possibility of hemicraniectomy. However, the patient's condition worsened, and the patient died on the third day after admission.

\section{DISCUSSION}

This study reported a case of MCA territory infarction in a patient with COVID-19. The incidence of cerebrovascular events in COVID-19 patients involving the MCA territory remains limited and undetailed. A similar case was found in a previous study that reported a 79-year-old male COVID-19 patient with new-onset mild ischemic stroke with ground-glass opacity on his chest CT results. $8,9 \mathrm{New}$-onset, large-vessel stroke in COVID-19 patients was also reported more recently in five patients younger than 50 years old from the United States. These patients are in a similar age group with the patient in this case. ${ }^{10}$
The initial symptoms of COVID-19, in this case, occurred 11 days after the patient had contact with another COVID-19 patient. In contrast, the MCA ischemic stroke occurred 3 days after the occurrence of the initial symptoms. The occurrence pattern of ischemic stroke in these patients was quite uncommon since several previous studies have shown an average range of 8 to 24 days in the ischemic stroke-related COVID-19 occurrence time. However, the previous study mentioned the possibility of developing an early occurrence of ischemic stroke in COVID-19. Hence, the mechanism of ischemic stroke induced by SARS-CoV-2 infection remains unclear. ${ }^{11}$

There is a report on how SARS-COV-2 infection leads to the occurrence of damaging immune reactions to the body. ${ }^{12}$ Reddy et $\mathrm{al}^{13}$ also stated the possibility that interleukin- 6 played an essential role in the occurrence of stroke cases in COVID-19 patients. Furthermore, a prior study also showed that inflammation also contributes to atherosclerosis and affects plaque stability. ${ }^{14}$ This study's results and hypothesis may explain why COVID-19 can provoke severe large artery vessel occlusion. In this case, signs of an inflammatory process were reported in the patient mainly because of SARS-CoV-2 infection. In addition, a recent study revealed that NLR had been identified as an independent risk factor for severe cases of COVID-19. Patients with age $\geq 50$ and NLR $\geq 3.13$ had a higher risk of developing severe illness and ICU admission. Similar to our case, the patient's age is 57 years, and NLR was 5 with poor outcome. ${ }^{15}$ 
A prior history of vascular comorbidities included hypertension and heart disease in this patient. These have been the main risk factors contributing to the patient's poor outcome. A retrospective study showed that age, underlying vascular diseases, and baseline NIHSS were predictors of poor outcome in patients with acute stroke. ${ }^{16}$

The patient had a hemorrhagic transformation after 48 hours of hospital admission. To this day, the pathophysiology of hemorrhagic transformation has not yet been established. However, several mechanisms might increase the chance of hemorrhagic transformation occurrence. In this patient, the possible mechanism was increased vascular permeability after the release of high intracranial pressure. The increases in brain permeability can occur because of prolonged ischemia and hypoxia in brain edema. ${ }^{17}$

It has already been reported that COVID-19 patients with thrombosis vascular events, including stroke, are more likely to develop acute respiratory distress syndrome, mechanical ventilation support, and intensive care admission. ${ }^{18}$ Another study also reported that the mortality rate of COVID-19 patients with large vessel occlusion reached 31\%, albeit when the optimal medication was given at the appropriate time. ${ }^{19}$ The previous study also discussed concern about the noticeable increase in the mortality rate of COVID-19 patients with stroke compared with other COVID-19 patients. In addition, evidence of systemic inflammation has been associated with increased CRP levels, D-dimer, and fibrin degradation products, leading to thrombotic vascular events. ${ }^{18}$

In COVID-19 patients, both D-dimer and high sensitivity (hs)-CRP levels were related to inflammation biomarkers. A recent study showed that in COVID-19 patients, the correlation between D-dimer levels and hs-CRP levels before treatments was related to increased levels of hs-CRP during disease progression. When the levels of hs-CRP exceeded $10 \mathrm{mg} / \mathrm{l}$, the correlation between D-dimer and hs-CRP was stronger. An elevation in D-dimer ( $>2.0 \mathrm{mg} / \mathrm{l})$ was associated with an increased risk of mortality and case fatality rate. These factors suggest that, besides inflammation, other factors were responsible for activating the coagulation system in patients with COVID-19. In our case, the CRP levels were elevated to $26 \mathrm{mg} / \mathrm{l}$, indicating an inflammatory process. However, the D-dimer and other coagulation tests were unable to be performed because the patient died shortly before further tests could be taken. ${ }^{15,20}$ All the above might imply that stroke contributes to the fatal outcomes in COVID-19 patients. However, the previous study reported that the challenge of estimating the contribution of a stroke to fatal outcomes, as most patients with stroke and SARS-CoV-2 infection were classified as severe COVID-19. ${ }^{18}$

Several conditions have to be considered explaining the poor outcomes of patients. One was the delayed access between symptom onset and arrival at the hospital led to a delay in patient management. This situation may have occurred because of increased difficulty transporting the patient related to the enactment of local lockdown policies and social distancing. Delays might also have been due to family members' concern and fear that the patient might become infected with SARS-COV-2 while seeking medical attention in the hospital. ${ }^{21}$

Our report has some limitations. First, we had limited access to workups and advanced diagnostic tools, therefore, we have limited evidence of other organ involvement that may affect these patients' outcomes. For example, a CT angiogram can provide a detailed location of the ischemic vessels and others. In this case, the patient's risk and the poor outcome could have been associated with the hypercoagulable state due to systemic inflammation, vascular comorbidities, and delayed hospital management access. Physicians must be aware of the potential incidence of cerebrovascular events in SARS-CoV-2 infection, especially in patients with risk factors.

In conclusion, MCA territory infarction was an unusual feature of COVID-19. Nevertheless, physicians should be aware of the underlying mechanism and possible risk factors associated with the possible incidence and predictors of cerebrovascular events in COVID-19 patients.

\section{Conflict of Interest}

The authors affirm no conflict of interest in this study.

\section{Acknowledgment}

We would like to thank the staff of Bethesda Hospital, Yogyakarta for their supports in this case report.

\section{Funding Sources}

None. 


\section{REFERENCES}

1. Li Q, Guan X, Wu P, Wang X, Zhou L, Tong Y, et al. Early transmission dynamics in Wuhan, China, of novel coronavirusinfected pneumonia. N Engl J Med. 2020;382(13)1199-207.

2. Huang C, Wang Y, Li X, Ren L, Zhao J, Hu Y, et al. Clinical features of patients infected with 2019 novel coronavirus in Wuhan, China. Lancet. 2020;395(10223):497-506.

3. Indonesian Task Force for COVID-19. Data of COVID-19 in Indonesia [Internet]. Indonesian Task Force for COVID-19; 2020 [cited $2020 \mathrm{Jul}$ 14]. Available from: https://covid19.go.id/petasebaran.

4. Pinzon RT, Wijaya VO, Buana RB, Al Jody A, Nunsio PN. Neurologic characteristics in coronavirus disease 2019 (COVID-19): a systematic review and meta-analysis. Front Neurol. 2020;11:565.

5. Walcott BP, Miller JC, Kwon CS, Sheth SA, Hiller M, Cronin CA, et al. Outcomes in severe middle cerebral artery ischemic stroke. Neurocrit Care. 2014;21(1):20-6.

6. Tunç A, Ünlübaş Y, Alemdar M, Akyüz E. Coexistence of COVID-19 and acute ischemic stroke report of four cases. J Clin Neurosci. 2020;77:227-9.

7. Trejo Gabriel y Galán JM. Stroke as a complication and prognostic factor of COVID-19 [Ictus comocomplicación y como factor pronóstico de COVID-19] Neurología (English Edition). 2020;35(5):318-22.

8. Belani P, Schefflein J, Kihira S, Rigney B, Delman BN, Mahmoudi $\mathrm{K}$, et al. COVID-19 is an independent risk factor for acute ischemic stroke. AJNR Am J Neuroradiol. 2020;41(8):1361-4.

9. Zhai P, Ding Y, Li Y. The impact of COVID-19 on ischemic stroke: a case report. Infect Dis [Preprint]. 2020 [cited 2020 May 25]. Available from: https://10.21203/rs.3.rs-20393/v1.

10. Oxley TJ, Mocco J, Majidi S, Kellner CP, Shoirah H, Singh IP, et al. Large-vessel stroke as a presenting feature of covid-19 in the young. N Eng J Med. 2020;382(60):e60.

11. Beyrouti R, Adams ME, Benjamin L, Cohen H, Farmer SF, Goh YY, et al. Characteristics of ischaemic stroke associated with COVID-19. J Neurol Neurosurg Psychiatry. 2020;91(8):889-91.

12. Zhang ZL, Hou YL, Li DT, Li FZ. Laboratory findings of COVID-19: a systematic review and meta-analysis. Scand J Clin Lab Invest. 2020;1-7.

13. Reddy ST, Garg T, Shah C, Nascimento FA, Imran R, Kan P, et al. Cerebrovascular disease in patients with COVID-19: a review of the literature and case series. Case Rep Neurol. 2020;12(2):199-209.

14. Hartmann P, Schober A, Weber C. Chemokines and microRNAs in atherosclerosis. Cell Mol Life Sc. 2015;72(17):3253-66.

15. Liu J, Liu Y, Xiang P, Pu L, Xiong H, Li C, et al. Neutrophil-tolymphocyte ratio predicts severe illness patients with 2019 novel coronavirus in the early stage. J Transl Med. 2020;18:206.

16. Wouters A, Nysten C, Thijs V, Lemmens R. Prediction of outcome in patients with acute ischemic stroke based on initial severity and improvement in the first 24 h. Front Neurol. 2018;9:308.

17. Zhang J, Yang Y, Sun H, Xing Y. Hemorrhagic transformation after cerebral infarction: current concepts and challenges. Ann Transl Med. 2014;2(8):81.

18. Tsivgoulis G, Katsanos AH, Ornello R, Sacco S. Ischemic stroke epidemiology during the COVID-19 pandemic: navigating uncharted waters with changing tides. Stroke. 2020;51(7):1924-6.

19. Sweid A, Hammoud B, Bekelis K, Missios S, Tjoumakaris SI, Gooch MR, et al. Cerebral ischemic and hemorrhagic complications of coronavirus disease 2019. Int J Stroke. 2020;1747493020937189.

20. Yu B, Li X, Chen J, Ouyang M, Zhang H, Zhao X, et al. Evaluation of variation in D-dimer levels among COVID-19 and bacterial pneumonia: a retrospective analysis. J Thromb Thrombolysis. 2020;1-10.

21. Zhao J, Li H, Kung D, Fisher M, Shen Y, Liu R. Impact of the COVID-19 epidemic on stroke care and potential solutions. Stroke. 2020;51:1996-2001. 